\title{
Nomogram-based prediction of survival in unresectable or metastatic gastric cancer patients with good performance status who received first-line chemotherapy
}

\author{
Jin Wang ${ }^{1,2}$, Bowen Yang ${ }^{1,2}$, Zhi $\mathrm{Li}^{1,2}$, Jinglei $\mathrm{Qu}^{1,2}$, Jing Liu ${ }^{1,2}$, Na Song ${ }^{1,2}$, Ying Chen ${ }^{1,2}$, Yu Cheng ${ }^{1,2}$, \\ Simeng Zhang ${ }^{1,2}$, Zhongqing Wang ${ }^{3}$, Xiujuan $\mathrm{Qu}^{1,2}$, Yunpeng Liu ${ }^{1,2}$ \\ ${ }^{1}$ Department of Medical Oncology, ${ }^{2}$ Key Laboratory of Anticancer Drugs and Biotherapy of Liaoning Province, ${ }^{3}$ Department of Information Center, \\ the First Hospital of China Medical University, Shenyang 110001, China \\ Contributions: (I) Conception and design: J Wang, X Qu, Y Liu; (II) Administrative support: X Qu, Y Liu; (III) Provision of study materials or \\ patients: J Qu, J Liu, N Song, Y Chen; (IV) Collection and assembly of data: Y Cheng, S Zhang, Z Wang; (V) Data analysis and interpretation: J \\ Wang, B Yang, Z Li; (VI) Manuscript writing: All authors; (VII) Final approval of manuscript: All authors. \\ Correspondence to: Yunpeng Liu; Xiujuan Qu. Professor, Department of Medical Oncology, the First Hospital of China Medical University, No.155 \\ Nanjing north street, Shenyang 110001, China. Email: ypliu@cmu.edu.cn; xiujuanqu@yahoo.com.
}

\begin{abstract}
Background: Good performance status (PS) is widely acknowledged to have a high prognostic ability, although the prognostic parameters of cancer patients with good PS are still uncertain. This study was conducted to establish and validate a point-based nomogram to assist with predicting prognosis in unresectable or metastatic gastric cancer (GC) patients who had good PS and underwent first-line chemotherapy.

Methods: At random, a total of 309 patients with GC were split into 2 cohorts: a training cohort $(\mathrm{n}=259)$ and an internal validation cohort $(\mathrm{n}=50)$. An independent external validation cohort comprising 147 patients was also recruited. Both univariate and multivariate Cox regression analyses were used to evaluate patients based on the overall survival (OS) to develop the nomogram, which was subsequently validated using the concordance index (c-index), calibration curve, and decision curve analysis (DCA).

Results: The nomogram contained 3 independent prognostic variables in the training cohort: the number of distant metastatic sites $(\mathrm{P}<0.001)$, carbohydrate antigen 199 (CA199) level $(\mathrm{P}=0.002)$, and fibrinogen $(\mathrm{P}=0.020)$. The nomogram predicted an OS with a c-index of 0.623 (95\% CI, 0.58-0.67) in the training cohort. The internal validation showed that the nomogram had a c-index of 0.614 (95\% CI, 0.51-0.72). For external validation, the c-index was 0.638 (95\% CI, 0.58-0.70).

Conclusions: A reliable point-based nomogram for predicting the prognosis of patients who had unresectable or metastatic GC and good PS who underwent first-line chemotherapy was developed and validated.
\end{abstract}

Keywords: Nomogram-based prediction; overall survival; unresectable gastric cancer; metastatic gastric cancer; good performance status; first-line chemotherapy

Submitted Oct 07, 2019. Accepted for publication Feb 04, 2020.

doi: $10.21037 /$ atm.2020.02.131

View this article at: http://dx.doi.org/10.21037/atm.2020.02.131

\section{Introduction}

Gastric cancer (GC) is the fourth most frequently diagnosed cancer in the world (1). In China, almost $80 \%$ of patients are diagnosed with advanced GC due to inadequate early diagnosis, and in contrast, the rates for patients in Japan and
South Korea are $<50 \%(2,3)$. For metastatic or recurrent GC, palliative chemotherapy is still the main treatment choice (4).

To date, several prognostic indicators have been reported to be associated with survival in metastatic or 


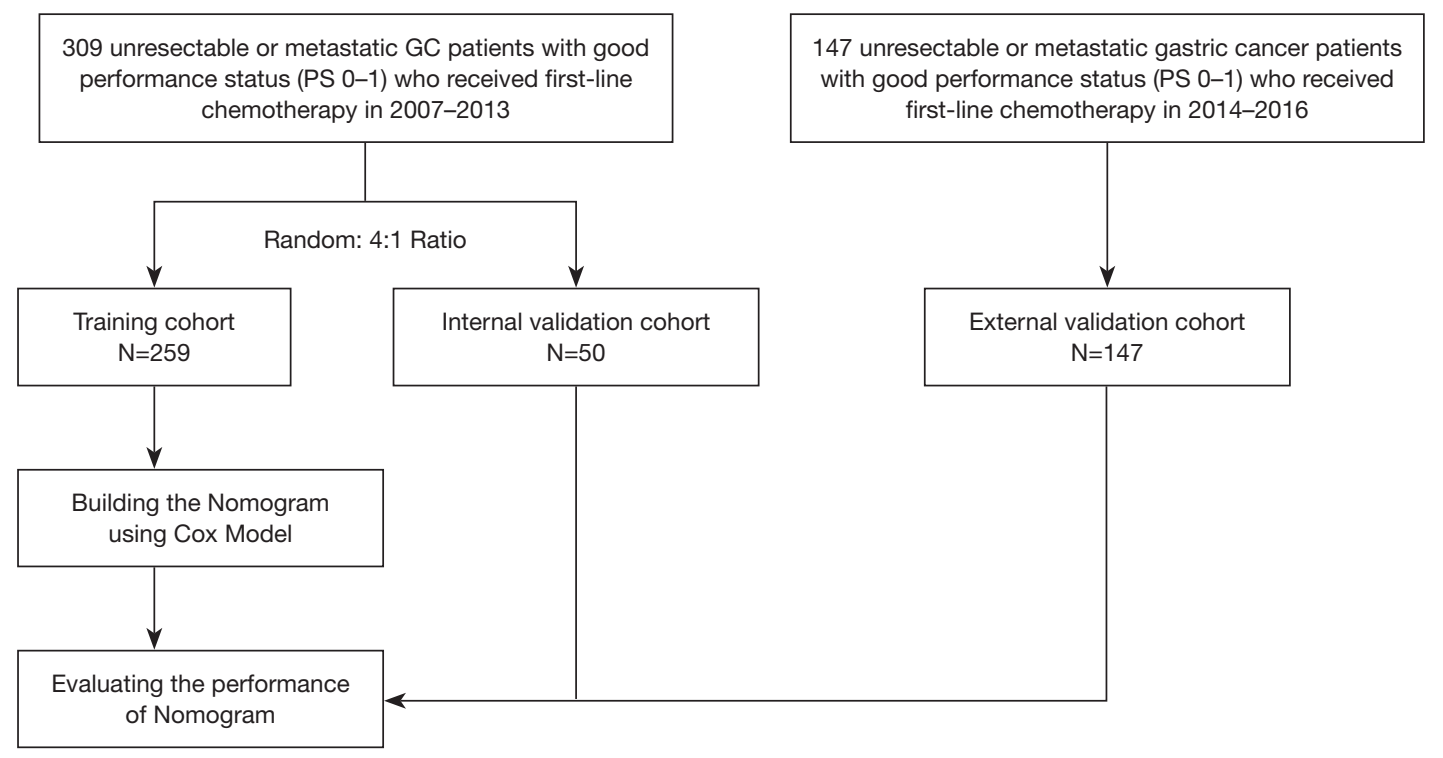

Figure 1 Flow chart of patient selection.

recurrent GC patients. Eastern Cooperative Oncology Group performance status (ECOG PS) is commonly used by physicians and nurses in oncology as it is regarded as an important prognostic factor for assessing treatment response and outcomes in cancer patients $(5,6)$. However, some cancer patients with good PS (0-1) status have poor prognosis clinically (7-9). Therefore, patients with good PS status might have some specific prognostic factors. To our knowledge, few studies have analyzed the prognostic parameters among this subset of cancer patients $(7,8,10,11)$. Although a model for the prognoses of patients with metastatic or recurrent GC and good PS who undergo chemotherapy as a first-line treatment has been modified before (7), some issues still need to be solved. Firstly, some important host- or tumor-related prognostic factors, such as coagulation markers and tumor biomarkers, that have been proved in the recent studies were not included in the previous prognostic model (12-15). Secondly, there is no validation procedure in the previous prognostic model (7). Thirdly, the previous prognostic models lacked the feature of integrating multiple putative prognostic factors into a single numerical evaluation (7,16-19). A points-based nomogram that differs from the traditional prognostic models could be an efficient tool by combining the prognostic factors that estimate survival probability tailored to individual patients (20).

We herein described the development, both internally and externally, of a nomogram to inform overall survival (OS) predictions for metastatic or recurrent GC patients with good performance status receiving first-line chemotherapy.

\section{Methods}

\section{Study design and population}

A retrospective cohort study was conducted on GC patients with good PS who received first-line palliative chemotherapy at the First Hospital of China Medical University. The inclusion criteria of patients were as follows: (I) aged $\geq 18$ years, (II) histologically confirmed diagnosis of GC, (III) with at least one measurable or evaluable lesion, (IV) who underwent at least one cycle of chemotherapy, (V) with PS 0-1, (VI) no clinical evidence of infection or other inflammatory conditions, and (VII) access to clinicopathological data at the beginning of chemotherapy. Those with esophageal cancer, squamous cell carcinomas, or gastroesophageal junction tumors were not eligible for inclusion. The study was in line with the Helsinki Declaration and approved by the Ethics Committee at the First Hospital of China Medical University (No. AFSOP-07-1.1-01). All patients have signed the informed consent form.

The flow chart for patient selection was shown in Figure 1. At random, a total of 309 patients (from April 2007 to December 2013) were split into 2 cohorts, a training cohort $(\mathrm{n}=259)$ and an independent internal validation cohort 
$(n=50)$, at a 4:1 ratio. An independent cohort with similar criteria for inclusion was used to validate our model, with subjects prospectively recruited between January 2014 and October 2016 ( $n=147$, the external validation cohort). The primary endpoint was OS, which was defined as the time from metastasis until death or the time of their ultimate follow-up visit.

\section{Nomogram development and construction}

To elaborate on the nomogram, 20 clinicopathological variables that are routinely measured based on published reports and authors' experience were considered. Featured in these variables were: (I) patient-based characteristics including age, gender, and ECOG PS; hematological parameters such as white blood cell (WBC) count, absolute neutrophil count (ANC), lymphocyte (LN) count, hemoglobin (HGB), platelet count (PLT), fibrinogen, total protein (TP), albumin (ALB), carcinoembryonic antigen (CEA), carbohydrate antigen 199 (CA199), neutrophil-tolymphocyte ratio (NLR), and platelet-to-lymphocyte ratio (PLR); (II) tumor-dependent variables such as previous history of gastrectomy, the number of distant metastatic sites, metastasis to the liver, bones, and lungs when chemotherapy commenced.

For developing a nomogram, univariate analyses of every clinicopathological variable were performed by way of a Cox proportional hazards regression model. The variables that achieved a significant level of $\mathrm{P}<0.2$ were entered into multivariate analyses for screening as independent risk factors via the Cox model with forwarding stepwise procedures. Based on all independent prognostic factors, a nomogram was constructed to allow for a visualized estimate of individual OS probability after 1 year.

\section{Nomogram validation}

Nomogram validation included 3 activities: firstly, 1,000 bootstrap replications were developed to estimate the Harrell's concordance index (c-index) in both pieces of training as well as validation cohorts (21). Secondly, to carry out calibration, we plotted the observed $v s$. predicted OS probabilities after one year, grouping all patients in the training and validation cohorts. Thirdly, the value of the nomogram with respect to competing benefits and problems was estimated using decision curve analysis (DCA).

\section{Statistical considerations}

Categorical variables were presented in the form of numbers and percentages. We recorded laboratory variables as continuous variables and presented them as mean \pm SD. The cut-off values for continuous variables were determined based on the normal value range. The clinicopathological parameters among the training cohort and the validation cohorts were analyzed using a Chi-square test or Fisher's exact test. We then conducted Cox regression analyses to evaluate independent prognostic factors in the training cohort. Statistical significance was considered to exist when $\mathrm{P}$ value $<0.05$, and all $\mathrm{P}$ values corresponded to significance tests (two-tailed). A receiver operating characteristic (ROC) curve analysis was carried out to calculate the area under the curve (AUC). SPSS 17.0 software package (SPSS, Chicago, IL) and R software, version 3.3.1 (http://www.r-project. org), facilitated the statistical analyses.

\section{Results}

\section{Baseline characteristics}

Overall, and in line with the eligibility criteria, a total of 259 patients were selected for the training cohort, 50 patients were selected for the internal validation cohort, and 147 patients were selected for the external validation cohort (Figure 1). Table 1 outlines the characteristics of patients across the two cohorts. The median OS for training and internal validation cohorts was 10.2 months [95\% confidence interval (CI), 9.30-10.94], and 12.1 months (95\% CI, 9.27-14.92), respectively. Patients who died at the time of the last follow-up in training and internal validation cohorts were $91.9 \%$ and $88 \%$, respectively. The external validation cohort had a median survival of 11.3 months $(95 \%$ CI, $9.85-12.81$ ), and $61.9 \%$ of patients died during the last follow-up visit.

\section{Prognostic nomogram}

Twenty clinicopathological variables were associated with OS in univariate analysis (Table 2). Subsequently, a multivariate Cox proportional hazards regression model was used in the training cohort (Table 3). Three covariates showed a significant correlation with survival: number of metastatic sites, fibrinogen, and CA199. Next, a prognostic nomogram was developed as shown in Figure 2, in which points were given based on the score of each variable of an individual 
Table 1 Characteristics of patients in the training and validation cohorts

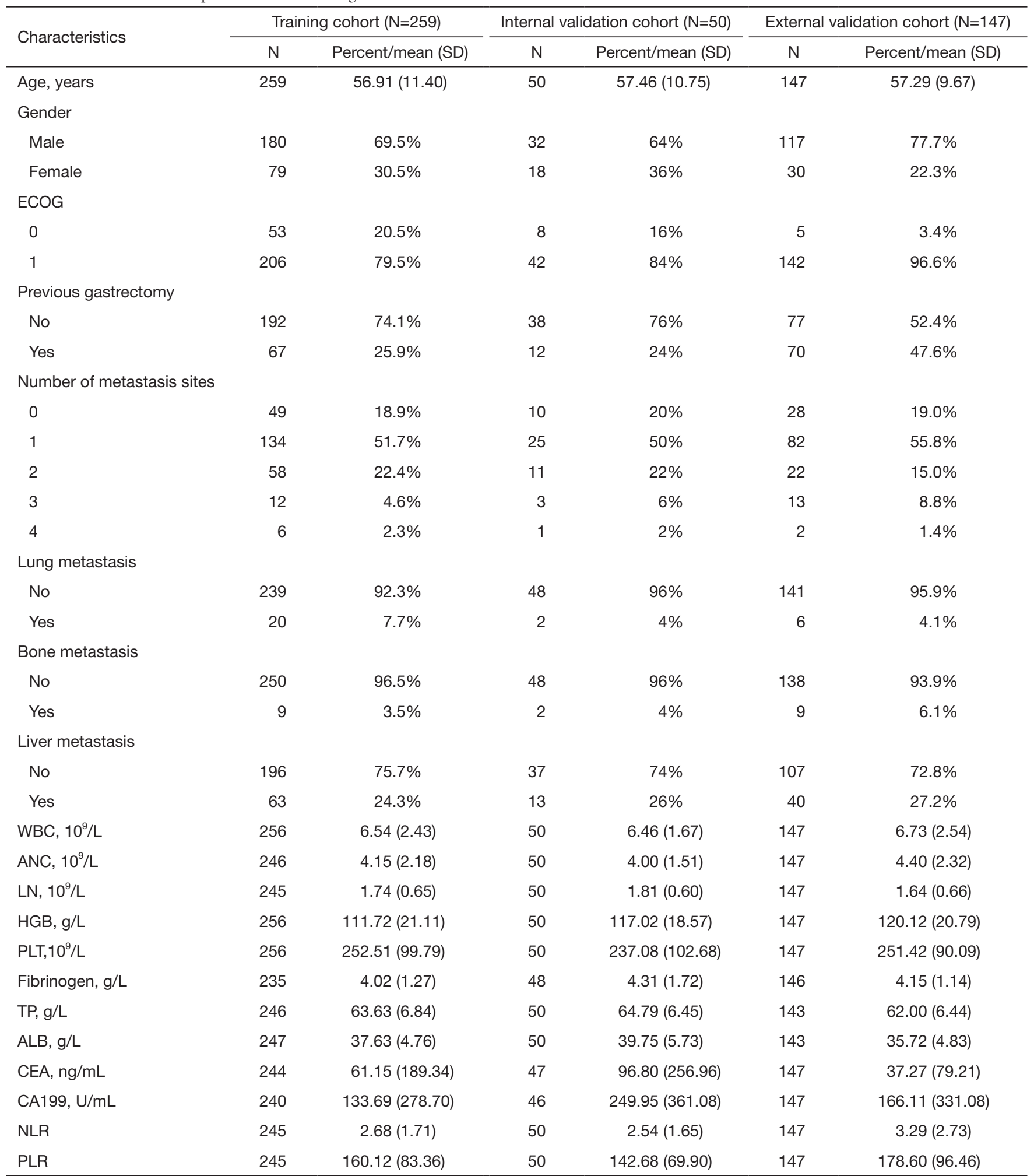

WBC, white blood cell count; ANC, Neutrophil count; LN, lymphocyte count; HGB, hemoglobin; PLT, platelet count; TP, total protein; ALB, albumin; CEA, carcinoembryonic antigen; CA199, carbohydrate antigen 199; NLR, neutrophil-to-lymphocyte ratio; PLR, platelet-tolymphocyte ratio. 
Table 2 Univariate analysis of clinical characteristics in relation to overall survival

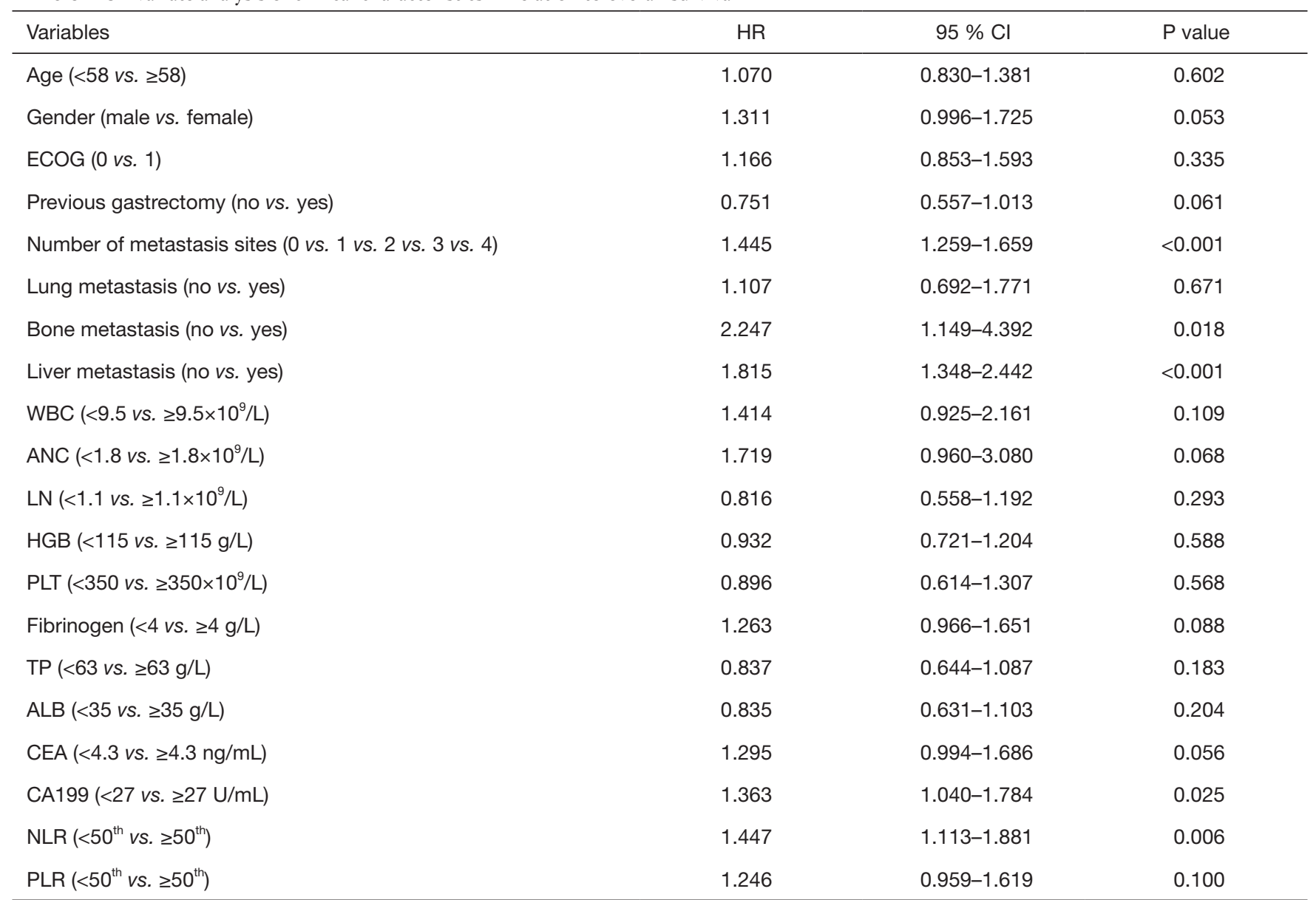

WBC, white blood cell count; ANC, Neutrophil count; LN, lymphocyte count; HGB, hemoglobin; PLT, platelet count; TP, total protein; ALB, albumin; CEA, carcinoembryonic antigen; CA199, carbohydrate antigen 199; NLR, neutrophil-to-lymphocyte ratio; PLR, platelet-tolymphocyte ratio.

Table 3 Multivariate analysis of clinical characteristics in relation to overall survival

\begin{tabular}{lccc}
\hline Variable & HR & $95 \% \mathrm{Cl}$ & $\mathrm{P}$ value \\
\hline Number of metastasis sites & 1.414 & $1.214-1.646$ & $<.001$ \\
Fibrinogen & 1.399 & $1.055-1.855$ & 0.020 \\
CA199 & 1.568 & $1.177-2.089$ & 0.002 \\
\hline
\end{tabular}

CA199, carbohydrate antigen 199.

patient by locating the corresponding scale of the variable. The total values were then summed up, and then a vertical line was drawn through the survival scales that provided the probability for 1-year OS. With the training cohort, the nomogram attained a bootstrap-corrected concordance index (c-index) of 0.623 (95\% CI, 0.58-0.67) (Figure 3A). Decision curve analysis (DCA) showed that if the threshold probability was $>0.6$, the developed nomogram was considered superior in predicting OS in all of the patients' dead scheme or none of the patients' dead scheme (Figure 3B). The ROC curve for predicting the OS showed a higher AUC (0.756) than the previous model (0.670) (Figure 3C). 


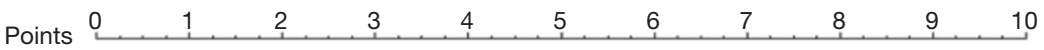
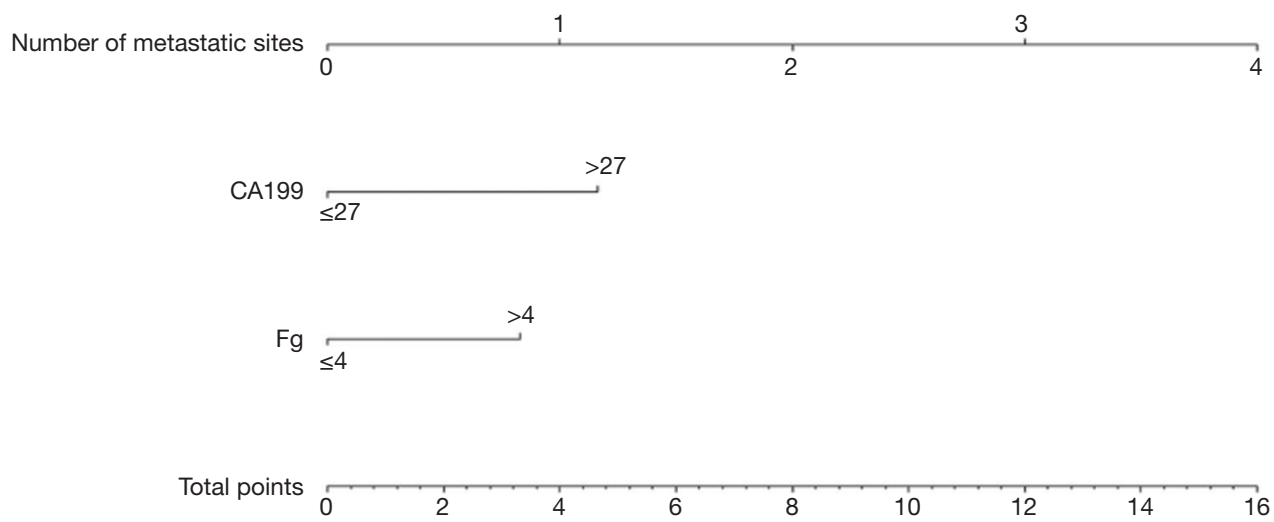

$\begin{array}{lllllll}\text { 1-year survival } & 0.6 & 0.5 & 0.4 & 0.3 & 0.2 & 0.1\end{array}$

Figure 2 Nomogram is predicting 1-year overall survival in patients who had unresectable or metastatic GC and good performance status who underwent first-line chemotherapy.
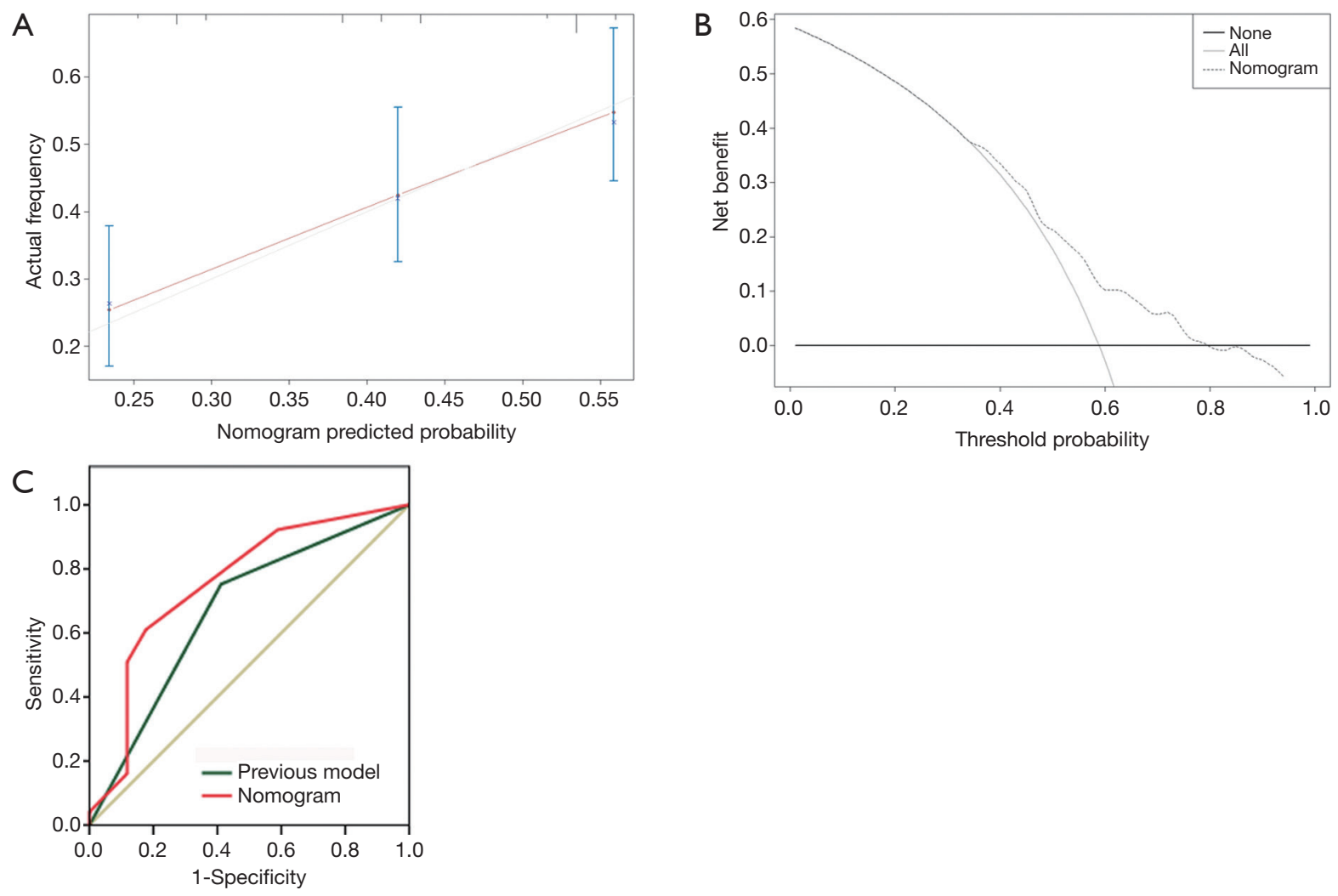

Figure 3 Evaluation of the nomogram in the training cohort. (A)The calibration plot was showing patient survival predictions after 1 year with a $95 \%$ confidence interval by decile (y-axis) overpredicted risk probability (x-axis) in the training cohort. A dashed line corresponds to a $10 \%$ margin of error. (B) The decision curve analysis (DCA) of the nomogram. (C) The ROC curves by nomogram and the previous model. 

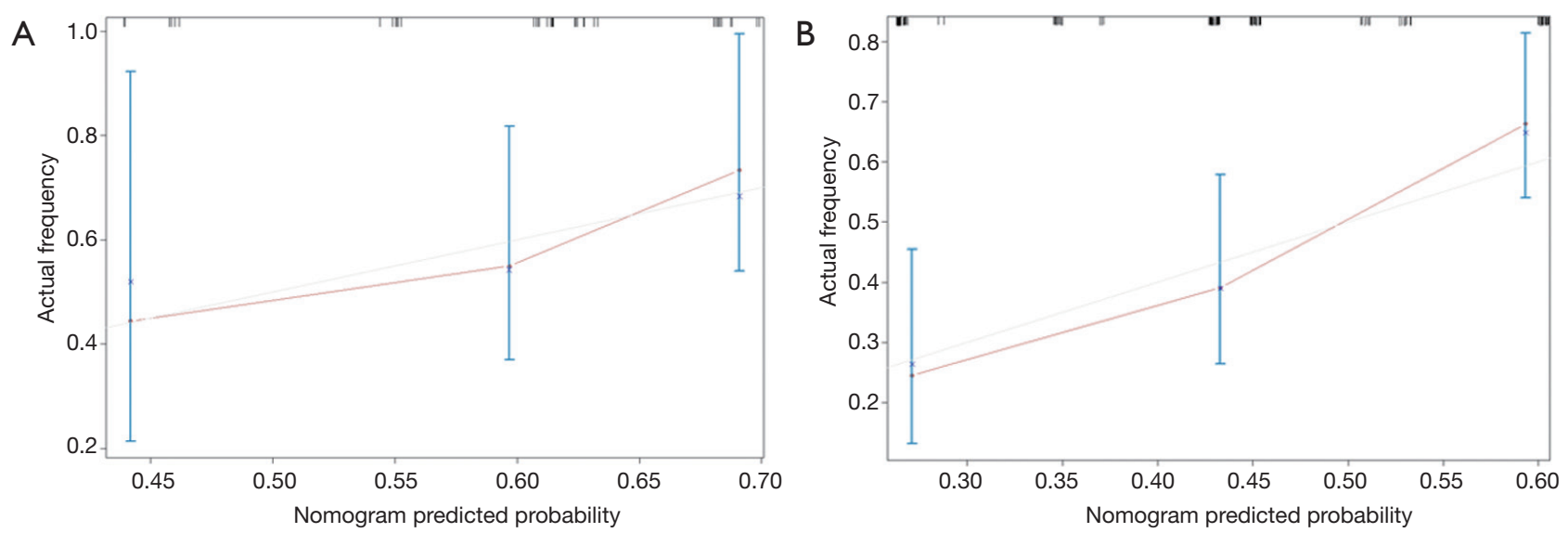

Figure 4 Validation of the nomogram. (A) The calibration plot is showing patient survival predictions after 1 year for the internal validation cohort; (B) the calibration plot was showing patient survival predictions after 1 year for the external validation cohort.

\section{Validation of the nomogram}

For the internal validation cohort, the nomogram achieved a c-index of 0.614 (95\% CI, 0.51-0.72). The plot that was calibrated displayed the 1-year OS probabilities between the predicted and actual survival (Figure 4A). When the nomogram was applied to the external validation, it demonstrated a c-index of 0.638 (95\% CI, 0.58-0.70) (Figure 4B).

\section{Discussion}

According to the nomograms in metastatic or recurrent GC patients who received first-line chemotherapy, only two studies have been reported $(22,23)$. However, there are some limitations still to address. The selection process for patients who underwent analysis in the previous nomograms, for example, was too indiscriminate, and patients with esophageal adenocarcinoma were included (23). In addition, some host inflammatory factors and tumor biomarkers that are prognostic clinicopathological factors were not involved in the nomograms analysis $(22,23)$. To our knowledge, no nomogram is available that includes host inflammatory factors, coagulation index, and tumor biomarkers for patients with metastatic or recurrent GC who have good PS and have received first-line chemotherapy.

The nomogram for individualized risk prediction could provide physicians as well as patients with a general outlook on prognosis from when GC is diagnosed (23). The final nomogram of our study consisted of three parameters, which included the number of metastatic sites, fibrinogen, and CA199. The number of metastatic sites, as a well-known factor, exhibited a significant negative prognostic effect in metastatic GC $(24,25)$. The patients with tumors were always in a hypercoagulable state, and thus coagulation index has been put forward as a key marker for the prognosis of different forms of cancer, including GC. Emerging evidence has indicated that a high level of plasma fibrinogen showed a significant association with poor OS in GC patients $(12,13)$. Our study for the first time, included fibrinogen in the development of a nomogram for metastatic GC. Fibrinogen acts as a scaffold molecule that affects the progression and metastasis of tumor cell growth and sustains the cellular responses of adhesion in different malignancies (26). Fibrinogen facilitates platelet adhesion in tumor cells, thereby protecting the tumor cells from escaping from the cytotoxicity of natural killer cells (27). Moreover, fibrinogen assessment might have implications for therapeutic optimization (27). According to a previous case report, systemic chemotherapy has successfully controlled the disseminated intravascular coagulation that is associated with advanced GC (28). The etiology and its contribution to therapeutic optimization still warranted further evaluation. Tumor markers that are easily obtained from the serum before chemotherapy was also evaluated in this study. Our nomogram showed CA199 as one of the independent risk factors. In recent studies, elevated serum CA199 was illustrated as an independent inferior prognostic factor in patients with metastatic GC $(14,15)$. The mechanism as to why CA199 affects the prognosis of patients with metastatic GC remains unclear. The reasons for the effects of CA199 were due to its role in intercellular adhesion and in cells that express this surface glycoprotein, which subsequently resulted in tumor metastasis and invasive potential (29). 
We previously modified a prognostic model for patients who have metastatic or recurrent GC and good PS (7). In the current study, the patient population was increased in 2016 compared to the 2013 model. In addition, coagulation markers, tumor biomarkers, and nutritional status markers (total protein and albumin) were added in the Cox regression analyses. Furthermore, a point-based nomogram with internal and external validation in which the previous model lacked was developed. More importantly, the ROC curve showed that the present nomogram had higher sensitivity and specificity for predicting OS than the previous model. This showed that the nomogram further optimized the model for the prognoses of patients who have metastatic or recurrent GC and good PS.

When considering the generalizability of the nomogram, there are some potential limitations that should be taken into account. First, we conducted the study retrospectively and designed it with a small population and a shorter duration of mean follow-up time. More patients are needed in both the training and validation cohorts. Secondly, the impact of subsequent lines of chemotherapy on the length of survival was not taken into account with our nomogram. Third, the human epidermal growth factor receptor 2 (HER2) status was not described in this study. Because the patients enrolled in the training cohort were from 2007 to 2013, and the HER2 status test in patients with metastatic GC was not a routine examination in the early years, especially before 2010, when Trastuzumab for Gastric Cancer (ToGA) study reported (30). Despite these limitations, it is worth noting that our nomogram had moderate discrimination ability for OS (c-index of 0.623 in the training cohort and 0.638 in the external validation cohort) and the calibration plots at 1 year were also good in all the cohorts.

\section{Conclusions}

In conclusion, a reliable nomogram was developed and validated to predict the OS of patients who had unresectable or metastatic GC and a good PS who underwent firstline chemotherapy. The developed nomogram has great potential for application in clinical practice for estimating the mortality risk in treating GC patients individually.

\section{Acknowledgments}

Funding: National Science and Technology Major Project of the Ministry of Science and Technology of China (No.2017ZX09304025); the National Natural
Science Foundation of China (No.81902998); Science and Technology Plan Project of Liaoning Province (No.2016007010); The General Projects of Liaoning Province Colleges and Universities (No.LFWK201706); Science and Technology Youth Projects of the Education Department of Liaoning Province (QN2019004).

\section{Footnote}

Conflicts of Interest: The authors have no conflicts of interest to declare.

Ethical Statement: The authors are accountable for all aspects of the work in ensuring that questions related to the accuracy or integrity of any part of the work are appropriately investigated and resolved. The study was in line with the Helsinki Declaration and approved by the Ethics Committee at the First Hospital of China Medical University (No.AF-SOP-07-1.1-01). Informed consent was obtained from the patients or their relatives.

Open Access Statement: This is an Open Access article distributed in accordance with the Creative Commons Attribution-NonCommercial-NoDerivs 4.0 International License (CC BY-NC-ND 4.0), which permits the noncommercial replication and distribution of the article with the strict proviso that no changes or edits are made and the original work is properly cited (including links to both the formal publication through the relevant DOI and the license). See: https://creativecommons.org/licenses/by-nc-nd/4.0/.

\section{References}

1. Siegel RL, Miller KD, Jemal A. Cancer statistics, 2020. CA Cancer J Clin 2020;70:7-30.

2. Nie $\mathrm{Y}, \mathrm{Wu} \mathrm{K}, \mathrm{Yu} \mathrm{J}$, et al. A global burden of gastric cancer: the major impact of China. Expert Rev Gastroenterol Hepatol 2017;11:651-61.

3. Yang K, Hu JK. Gastric cancer treatment: similarity and difference between China and Korea. Transl Gastroenterol Hepatol 2017;2:36.

4. Yano T, Wang KK. Photodynamic Therapy for Gastrointestinal Cancer. Photochem Photobiol 2019. [Epub ahead of print].

5. Bersanelli M, Brighenti M, Buti S, et al. Patient performance status and cancer immunotherapy efficacy: a meta-analysis. Med Oncol 2018;35:132.

6. Ben Kridis W, Marrekchi G, Mzali R, et al. Prognostic factors in metastatic gastric carcinoma. Exp Oncol 
2019;41:173-5.

7. Penel N, Vanseymortier M, Bonneterre ME, et al. Prognostic factors among cancer patients with good performance status screened for phase I trials. Invest New Drugs 2008;26:53-8.

8. Wang J, Qu J, Li Z, et al. A Prognostic Model in Metastatic or Recurrent Gastric Cancer Patients with Good Performance Status Who Received First-Line Chemotherapy. Transl Oncol 2016;9:256-61.

9. Takahashi M, Takahashi M, Komine K, et al. The G8 screening tool enhances prognostic value to ECOG performance status in elderly cancer patients: A retrospective, single institutional study. PLoS One 2017;12:e179694.

10. Moriwaki T, Ishige K, Araki M, et al. Glasgow Prognostic Score predicts poor prognosis among advanced biliary tract cancer patients with good performance status. Med Oncol 2014;31:287.

11. Abrao FC, Peixoto RD, de Abreu IR, et al. Prognostic factors in patients with malignant pleural effusion: Is it possible to predict mortality in patients with good performance status? J Surg Oncol 2016;113:570-4.

12. Woo Y, Goldner B, Son T, et al. Western Validation of a Novel Gastric Cancer Prognosis Prediction Model in US Gastric Cancer Patients. J Am Coll Surg 2018;226:252-8.

13. Liu X, Liu Z, Lin E, et al. A cumulative score based on preoperative fibrinogen and the neutrophil-lymphocyte ratio to predict outcomes in resectable gastric cancer. Cancer Manag Res 2018;10:3007-14.

14. Brenkman HJF, Cho M, Ruurda JP, et al. European validation of the Yonsei Gastric Cancer Prognosis Prediction Model after gastrectomy: Validation with the Netherlands Cancer Registry. Eur J Surg Oncol 2019;45:983-8.

15. Yamamoto M, Kurokawa Y, Kobayashi N, et al. Prognostic Value of the Combined Index of Plasma Fibrinogen and the Neutrophil-Lymphocyte Ratio in Gastric Cancer. World J Surg 2020;44:207-12.

16. Fanotto V, Cordio S, Pasquini G, et al. Prognostic factors in 868 advanced gastric cancer patients treated with second-line chemotherapy in the real world. Gastric Cancer 2017;20:825-33.

17. Fuchs CS, Muro K, Tomasek J, et al. Prognostic Factor Analysis of Overall Survival in Gastric Cancer from Two Phase III Studies of Second-line Ramucirumab (REGARD and RAINBOW) Using Pooled Patient Data. J Gastric Cancer 2017;17:132-44.

18. Hsu JT, Liao JA, Chuang HC, et al. Palliative gastrectomy is beneficial in selected cases of metastatic gastric cancer. BMC Palliat Care 2017;16:19.

19. Richter AN, Khoshgoftaar TM. A review of statistical and machine learning methods for modeling cancer risk using structured clinical data. Artif Intell Med 2018;90:1-14.
20. Kim SY, Yoon MJ, Park YI, et al. Nomograms predicting survival of patients with unresectable or metastatic gastric cancer who receive combination cytotoxic chemotherapy as first-line treatment. Gastric Cancer 2018;21:453-63.

21. Custodio A, Carmona-Bayonas A, Jimenez-Fonseca P, et al. Nomogram-based prediction of survival in patients with advanced oesophagogastric adenocarcinoma receiving first-line chemotherapy: a multicenter prospective study in the era of trastuzumab. Br J Cancer 2017;116:1526-35.

22. Narita Y, Kadowaki S, Oze I, et al. Establishment and validation of prognostic nomograms in first-line metastatic gastric cancer patients. J Gastrointest Oncol 2018;9:52-63.

23. van den Boorn HG, Abu-Hanna A, Ter Veer E, et al. SOURCE: A Registry-Based Prediction Model for Overall Survival in Patients with Metastatic Oesophageal or Gastric Cancer. Cancers (Basel) 2019;11. doi: 10.3390/ cancers11020187.

24. Repetto O, De Re V. Coagulation and fibrinolysis in gastric cancer. Ann N Y Acad Sci 2017;1404:27-48.

25. Zheng S, Shen J, Jiao Y, et al. Platelets and fibrinogen facilitate each other in protecting tumor cells from natural killer cytotoxicity. Cancer Sci 2009;100:859-65.

26. Lee DS, Yoo SJ, Oh HS, et al. Advanced Gastric Cancer Associated with Disseminated Intravascular Coagulation Successfully Treated with 5-fluorouracil and Oxaliplatin. J Gastric Cancer 2013;13:121-5.

27. Okubo Y, Kawasaki Y, Tono T, et al. [CA19-9 Producing Gastric Cancer with Early Recurrence of Multiple Colon Metastases Following Gastrectomy-A Case Report]. Gan To Kagaku Ryoho 2018;45:2447-9.

28. Huang C, Liu Z, Xiao L, et al. Clinical Significance of Serum CA125, CA19-9, CA72-4, and Fibrinogen-toLymphocyte Ratio in Gastric Cancer With Peritoneal Dissemination. Front Oncol 2019;9:1159.

29. Magalhães A, Duarte HO, Reis CA. Aberrant Glycosylation in Cancer: A Novel Molecular Mechanism Controlling Metastasis. Cancer Cell 2017;31:733-5.

30. Bang YJ, Van Cutsem E, Feyereislova A, et al. Trastuzumab in combination with chemotherapy versus chemotherapy alone for treatment of HER2-positive advanced gastric or gastro-oesophageal junction cancer (ToGA): a phase 3, open-label, randomised controlled trial. Lancet 2010;376:687-97.

Cite this article as: Wang J, Yang B, Li Z, Qu J, Liu J, Song N, Chen Y, Cheng Y, Zhang S, Wang Z, Qu X, Liu Y. Nomogrambased prediction of survival in unresectable or metastatic gastric cancer patients with good performance status who received first-line chemotherapy. Ann Transl Med 2020;8(6):311. doi: 10.21037/atm.2020.02.131 